\title{
Argus++: Robust Real-time Activity Detection for Unconstrained Video Streams with Overlapping Cube Proposals
}

\author{
Lijun Yu, Yijun Qian, Wenhe Liu, and Alexander G. Hauptmann \\ Language Technologies Institute, Carnegie Mellon University \\ \{lijun, yijunqian@cmu.edu\}, \{wenhel, alex\}@cs.cmu.edu
}

\begin{abstract}
Activity detection is one of the attractive computer vision tasks to exploit the video streams captured by widely installed cameras. Although achieving impressive performance, conventional activity detection algorithms are usually designed under certain constraints, such as using trimmed and/or object-centered video clips as inputs. Therefore, they failed to deal with the multi-scale multi-instance cases in real-world unconstrained video streams, which are untrimmed and have large field-of-views. Real-time requirements for streaming analysis also mark brute force expansion of them unfeasible.

To overcome these issues, we propose Argus++, a robust real-time activity detection system for analyzing unconstrained video streams. The design of Argus ++ introduces overlapping spatio-temporal cubes as an intermediate concept of activity proposals to ensure coverage and completeness of activity detection through over-sampling. The overall system is optimized for real-time processing on standalone consumer-level hardware. Extensive experiments on different surveillance and driving scenarios demonstrated its superior performance in a series of activity detection benchmarks, including CVPR ActivityNet ActEV 2021, NIST ActEV SDL UF/KF, TRECVID ActEV 2020/2021, and ICCV ROAD 2021.
\end{abstract}

\section{Introduction}

Nowadays, activity detection has drawn a fast-growing attention in both industry and research fields. Activity detection in extended videos [4, 15] is widely applied for public safety in indoor and outdoor scenarios. Activity detection on streaming videos captured by in-vehicle cameras is applied for vision-based autonomous driving. The development of these applications brings several challenges. First, most of these systems take unconstrained videos as input, which are recorded in large field-of-views where multi-object and multi-activity occur simultaneously and continuously over time. Second, the unconstrained videos in real world are in multiple scenarios and under multiple conditions, e.g. in dynamically changed road environments from day to night in autonomous driving [21]. Third, efficient algorithms are demanded for real-time processing and responding of streaming video.

Conventional activity detection works [22, 6, 23, 10, 7] have achieved impressive performance. However, they are not suitable for real world unconstrained video understanding. Most of these works are applied under certain constrains, e.g., only for processing trimmed and/or object-centered video clips. Meanwhile, they usually are specified for certain scenarios, such as person activity, etc. Therefore, such algorithms would fail when being transferred to unconstrained videos on both efficiency and effectiveness.

Previous works [20, 29, 13] on unconstrained video analysis proposed to generate and analyze tube/tubelet proposals, which are trajectories extracted from object detection and tracking results. Tube proposal has several drawbacks. First, tube proposals failed to capture the trace of moving objects when cropping the proposals from the original videos. Therefore, learning the activities highly relied on trace would be difficult, e.g. 'vehicle turning right'. Second, the tube proposals still cannot stay away from temporal activity localization to determine the existence of the activities. Besides, most of the previous works [20] utilize non-overlapping proposals, which straightforwardly cuts the tube proposals by fixed length of temporal windows. Inevitably, such methods destroy the completeness of activities. Therefore, it would result in significant degrade of performance. Third, the objects in the tube proposal will suffer from the bounding box shift and distortion across frames, which could result in a high false alarm rate on activity detection.

To overcome the aforementioned challenges, we propose Argus ++, an efficient robust spatio-temporal activity detec- 


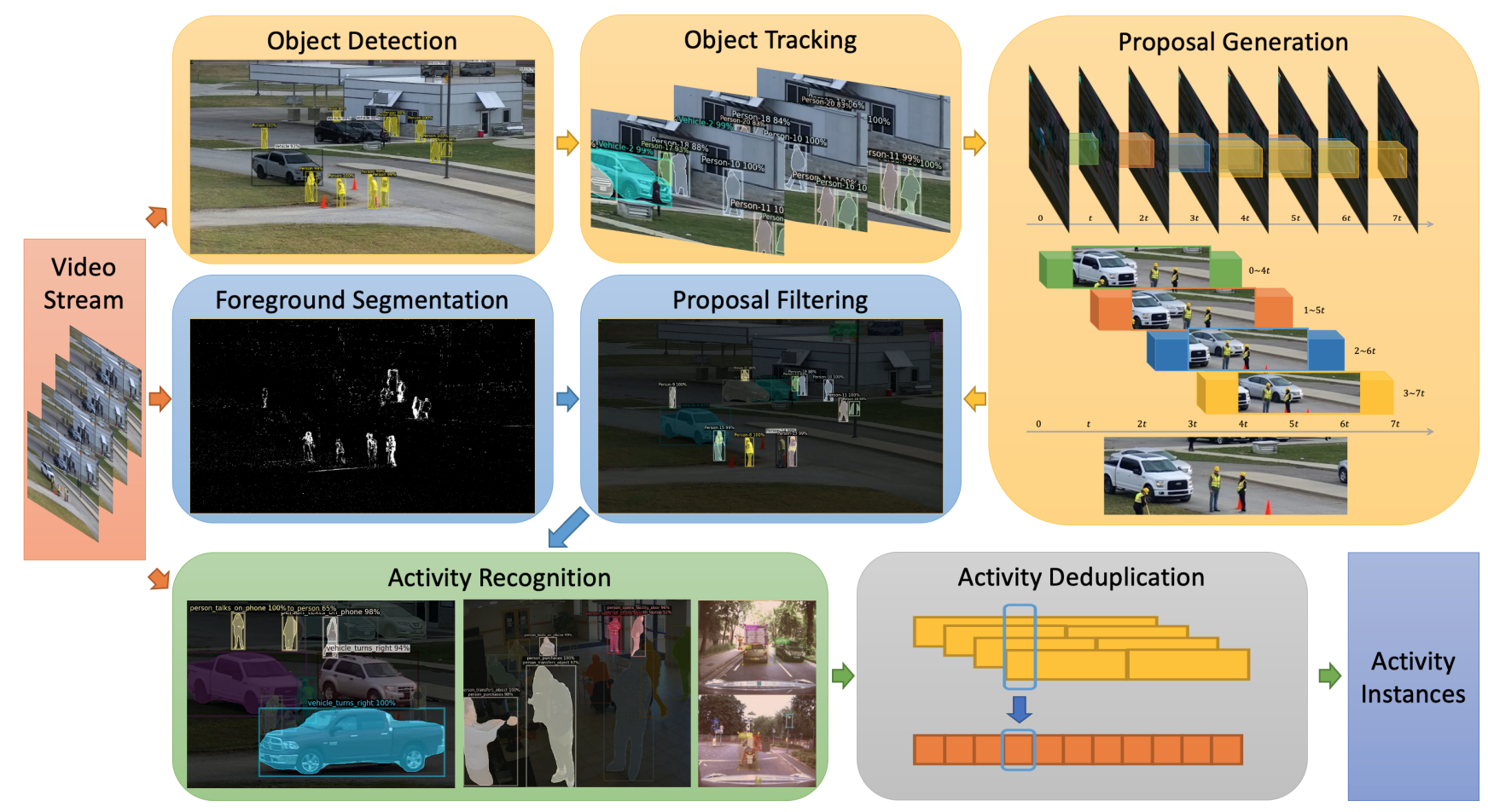

Figure 1. Architecture of Argus++. A video stream is processed frame-by-frame through object detection and tracking to generate overlapping cube proposals. With frame-level foreground segmentation, stable proposals are filtered out. Activity recognition models determine the classification scores for each proposal. These over-sampled cubes are deduplicated to produce the final activity instances.

tion system for extended and road video activity detection. The proposed system contains four-stages: Proposal Generation, Proposal Filtering, Activity Recognition and Activity Deduplication. The major difference between Argus++ and the former works, such as [13], is the concept of cube proposals. Rather than simply adapted tube proposals, i.e. cropped trajectories of detected and tracked objects, we propose to merge and crop the area of detected objects across the frames.

We summarize the contributions of our work as follows:

1. We propose Argus++, a real-time activity detection system for unconstrained video streams, which is robust across different scenarios.

2. We introduce overlapping spatio-temporal cubes as the core concept of activity proposals to ensure coverage and completeness of activity detection through oversampling.

3. The proposed system has achieved outstanding performance in a large series of activity detection benchmarks, including CVPR ActivityNet ActEV 2021, NIST ActEV SDL UF/KF, TRECVID ActEV 2020/2021, and ICCV ROAD 2021.

\section{Related Work}

Object Detection and Tracking Object detection and tracking are fundamental computer vision tasks that aims to detect and track objects from images or videos. Image-based object detection algorithms, such as Faster R-CNN [19] and R-FCN [5], have demonstrated convincing performance but are often expensive to apply on every frame. Video-based object detection algorithms [32, 18] use optical flow guided feature aggregation to leverage motion information and reduce computation. With the deep features extracted from the backbone convolutional network, multi-object tracking algorithms [25, 24] associates objects across frames based on feature similarity and location proximity.

Activity Detection In recent years, there emerged some systems designed for spatio-temporal activity detection on unconstrained videos [20, 29, 13, 3, 27, 31]. Generally, theses systems first generates activity proposals and then feeds them to classification models. Since there have been a variety of video classification networks [22, 11, 6], the major focus is on the paradigm of proposals and the generation algorithm. In [13, 3], a detection and tracking framework is employed to extract whole object tracklets as tubelets, where temporal localization is required. In [20], an encoderdecoder network is used to generate localization masks on fixed-length clips for tubelet proposal extraction, which has varied spatial locations in different frames. 


\section{Method}

\subsection{Activity Detection Task}

In this paper, we tackle the activity detection task in unconstrained videos which are untrimmed and with large field-of-views. Given an untrimmed video stream $\mathcal{V}$, the system $\mathcal{S}$ should identify a set of activity instances $\mathcal{S}(\mathcal{V})=$ $\left\{A_{i}\right\}$. Each activity instance is defined by a three-tuple $A_{i}=\left(T_{i}, L_{i}, C_{i}\right)$, referring to an activity of type $C_{i}$ occurs at temporal window $T_{i}$ with spatial location $L_{i} . L_{i}$ contains the precise location of $A_{i}$ in each frame, forming a tube in the timeline. As such, activity detection can often be decomposed into three aspects, i.e., temporal localization $\left(T_{i}\right)$, spatial localization $\left(L_{i}\right)$, and action classification $\left(C_{i}\right)$.

Each of the three aspects poses unique challenges to the video understanding system. Due to its multi-dimensional nature, it remains hard to define and build a useful activity detection system under the strict setting. Therefore, we also evaluates with some loosened requirements.

Strict Setting All activity types are defined as atomic activities with clear temporal boundaries and spatial extents. The evaluation metric performs bipartite matching between predictions and ground truths.

Loosened Setting Activity types are either atomic activities within a temporal window (e.g. standing up) or continuous repetitive activities that can be cut into multiple identifiable windows (e.g. walking). The evaluation metric allows multiple non-overlapping predictions to be matched with one ground truth.

\subsection{Argus++ System}

The architecture of the proposed Argus++ system is shown in Figure 1 . To tackle the task of activity detection, we adopt an intermediate concept of spatio-temporal cube proposal with a much simpler definition than an activity instance:

$$
p_{i}=\left(x_{0}^{i}, x_{1}^{i}, y_{0}^{i}, y_{1}^{i}, t_{0}^{i}, t_{1}^{i}\right)
$$

This six-tuple design relieves the localization precision and caters modern action classification models which works on fixed-length clips with fixed spatial window.

For an input video stream, the system first generates candidate proposals with frame-wise information such as detected objects, which will be covered in Section 3.3. These proposals are filtered with a background subtraction model as detailed in Section 3.4. Then, action recognition models described in Section 3.5 are applied on the proposals to predict per-class confidence scores. Finally, Section 3.6 introduces the post-processing stage to merge and filter the proposals with scores and generate final activity instances.

\subsection{Proposal Generation}

Starting this section, we introduce each of the components of Argus ++. The system begins by generating a set of cube proposals. They are generated based on information from frame-level object detection with multiple object tracking methods. Cubes are sampled densely in the timeline with refined spatial locations.

Detection and Tracking To conduct activity recognition, we first locate the candidate objects (in most cases, person and vehicle) in the video. For each selected frame $F_{i}$, we apply an object detection model to get objects $O_{i}=\left\{o_{i, j} \mid\right.$ $\left.j=1, \cdots, n_{i}\right\}$ with object types $c_{i, j}$ and bounding boxes $\left(x_{0}, x_{1}, y_{0}, y_{1}\right)_{i, j}$. Objects are detected in a stride of every $S_{d e t}$ frames. A multiple object tracking algorithm is applied on the detected objects to assign track ids to each of them as $t r_{i, j}$.

Proposal Sampling To sample proposals on untrimmed videos without breaking the completeness of any activity instances, we propose a dense overlapping proposals sampling algorithm. As illustrated in Figure 2, this method ensures coverage of activities occurring at any time, with no hard boundaries. Two parameters, duration $D_{\text {prop }}$ and stride $S_{\text {prop }}$, controls the sampling process. Each proposal contains a temporal window of $D_{\text {prop }}$ frames. New proposals are generated every $S_{\text {prop }} \leq D_{\text {prop }}$ frames, possibly with overlaps. Generally, non-overlapping proposal system can be treated as a degraded case when $S_{\text {prop }}=D_{\text {prop }}$.

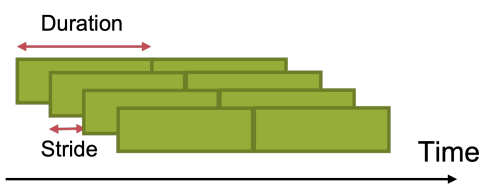

Figure 2. Dense Overlapping Proposals

Proposal Refinement To generate proposals in a temporal window from $t_{0}$ to $t_{1}=t_{0}+D_{\text {prop }}$, we select seed track ids $\operatorname{Tr}_{t_{c}}$ from the central frame $t_{c}=\left\lfloor\frac{t_{0}+t_{1}}{2}\right\rfloor$. Their bounding boxes are enlarged as the union across the temporal window

$$
\begin{gathered}
\left(x_{0}, x_{1}, y_{0}, y_{1}\right)_{k}=\bigcup\left(\left\{\left(x_{0}, x_{1}, y_{0}, y_{1}\right)_{i, j} \mid\right.\right. \\
\left.\left.t_{0} \leq i \leq t_{1}, t r_{i, j}=t r_{t_{c}, k}\right\}\right) \\
k=1, \cdots, n_{t_{c}}
\end{gathered}
$$

This algorithm is robust through identity switch in the tracking algorithm as it uses the stable seeds from the central frame. It also ensures the coverage of moving objects by enlarging the bounding box when it's successfully tracked. This design is helpful for efficiency optimization by allowing 

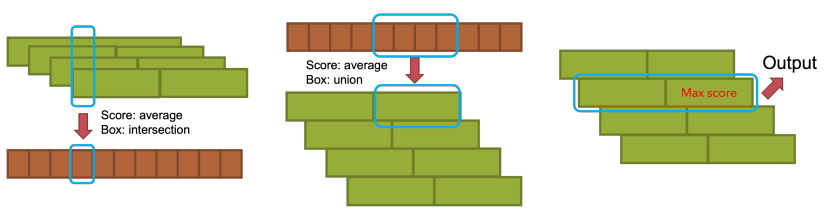

Figure 3. Deduplication Algorithm for Overlapping Proposals

a large detection stride $S_{\text {det }}$. When later applied for activity recognition, the bounding box can be further enlarged for a fixed rate $R_{e n l}$ to include spatial context and compensate for missed tracks.

\subsection{Proposal Filtering}

For now, the proposal generation pipeline applies a framewise object detection with slight aid of tracking information. The motion information of video is not yet explored. To produce high quality proposals, we apply a proposal filtering algorithm to eliminate the proposals that are unlikely to contain activities.

Foreground Segmentation For each proposal, a foreground segmentation algorithm is implemented to generate a binary mask for every $S_{b g}$ frames for each video clip. We average the value of pixel masks in its cube to get its foreground score $f_{i}$. For proposals generated by object type $c$, those proposals with $f_{i} \leq F_{c}$ will be filtered out. The threshold $F_{c}$ is determined by allowing up to $P_{\text {pos }}$ true proposals to be filtered out.

Label Assignment To determine the above threshold and to train the activity recognition module, we need to assign labels for each generated proposal according to the ground truth activity instances. We first convert the annotation of activity instances into the cube format, denoted as ground truth cubes, by performing dense sampling of duration $D_{\text {prop }}$ and stride $S_{\text {prop }}$ within each instance. For each proposal, we estimate the spatial intersection-over-union (IoU) between it and ground truth cubes in the same temporal window. Then we follow Faster R-CNN [19] in the assignment process:

- For each ground truth cube, assign it to the proposal with the highest score above $S_{\text {low }}$.

- For each proposal, assign it with each ground truth cube with score above $S_{\text {high }}$.

- For each proposal, assign it as negative if all scores are below $S_{\text {low }}$.

$S_{\text {high }}$ and $S_{\text {low }}$ are the high and low thresholds. Through this algorithm, each proposal may be assigned one or more positive labels, a negative label, or nothing. Those assigned nothing are redundant detections which will not be used in classifier training.
Proposal Evaluation To measure the quality of proposals before and after the filtering, we need a method for proposal evaluation. This can be achieved by assuming a perfect classifier in the activity recognition part, so the final metrics reflects the upper bound performance with current proposals. To do this, we simply use the assigned labels as the classification outputs and pass through the deduplication algorithm covered later. To further measure other properties of the generated proposals, we can only pass through a subset of them, such as only those with spatial IoU against ground truth above 0.5 .

\subsection{Activity Recognition}

In this section, we will elaborately introduce our action recognition modules. Given the input proposal of an activity instance $p_{i}$, our action recognition model $\mathbb{V}$ will give out the confidence vector $c_{i}$ :

$$
\mathbb{V}\left(p_{i}\right)=c_{i}=\left\{c_{i}^{1}, c_{i}^{2}, \ldots c_{i}^{n}\right\}
$$

Where $\mathrm{n}$ represents the number of target actions, and $c_{i} \in$ $\mathbb{R}^{n}$. Limited by GPU memory size and temporal length settings of pretrained weights, we need to select $t$ frames out of $t_{1}^{i}-t_{0}^{i}$ samples from the activity instance. To do this, we strictly followed the sparse-sampling strategy mentioned in [23] for both training and inference stage. To be specific, the video is evenly separated into $t$ segments. From each segment, 1 frame will be randomly selected to generate the sampled clip.

To transform the action recognition modules from previous multi-class task to the realm of multi-label recognition, we modified the loss function for optimization. Instead of traditional cross entropy loss (XE), we implemented a weighted binary cross entropy loss (wBCE). In which, two weight parameters are adopted, the activity-wise weight $\mathrm{W}_{a}=\left\{w_{a}^{1}, w_{a}^{2}, \ldots, w_{a}^{n}\right\}$ and the positive-negative weight $\mathrm{W}_{p}=\left\{w_{p}^{1}, w_{p}^{2}, \ldots, w_{p}^{n}\right\} . \mathrm{W}_{a}$ balances the training samples of different activities and $\mathrm{W}_{p}$ balances the positive and negative samples of a specific activity. With the aligned label sequence of $i^{t h}$ instance represented as $Y_{i}=\left\{y_{i}^{1}, y_{i}^{2}, \ldots, y_{i}^{n}\right\} \in \mathbb{R}^{n}$. The calculation of $w_{a}^{c}$ is derived as:

$$
\begin{gathered}
\hat{w}_{a}^{c}=\frac{1}{\sum_{i \in[I]} y_{i}^{c}} \\
w_{a}^{c}=n \times \frac{\hat{w}_{a}^{c}}{\sum_{c \in[n]} \hat{w}_{a}^{c}}
\end{gathered}
$$

And the derivation of $w_{p}^{c}$ is:

$$
w_{p}^{c}=\frac{\sum_{i \in[I]} \mathbf{1}_{y_{i}^{c}=0}}{\sum_{i \in[I]} y_{i}^{c}}
$$

1 http://activity-net.org/challenges/2021/ challenge.html

$\sqrt[2]{\text { https://actev.nist.gov/sdl\#tab_leaderboard }}$ 
Table 1. CVPR 2021 ActivityNet Challeng $\AA^{1}$ ActEV SDL Unknown Facility Evaluation

\begin{tabular}{lccc}
\hline System/Team & $n A U D C @ 0.2 T_{f a} \downarrow$ & Mean $P_{\text {miss }} @ 0.02 T_{f a} \downarrow$ & Relative Processing Time \\
\hline Argus++ (Ours) & $\mathbf{0 . 3 5 3 5}$ & $\mathbf{0 . 5 7 4 7}$ & 0.576 \\
UMD_JHU & $\underline{0.4232}$ & 0.6250 & 0.345 \\
IBM-Purdue & 0.4238 & 0.6286 & 0.530 \\
UCF & 0.4487 & $\underline{0.5858}$ & 0.615 \\
Visym Labs & 0.4906 & 0.6775 & 0.770 \\
MINDS_JHU & 0.6343 & 0.7791 & 0.898 \\
\hline
\end{tabular}

Table 2. NIST ActEV'21 SDL ${ }^{2}$ Known Facility Evaluation

\begin{tabular}{lccc}
\hline System/Team & $n A U D C @ 0.2 T_{f a} \downarrow$ & Mean $P_{\text {miss }} @ 0.02 T_{f a} \downarrow$ & Relative Processing Time \\
\hline Argus++ (Ours) & $\mathbf{0 . 1 6 3 5}$ & $\mathbf{0 . 3 4 2 4}$ & 0.413 \\
UCF & $\underline{0.2325}$ & $\underline{0.3793}$ & 0.751 \\
UMD & 0.2628 & 0.4544 & 0.380 \\
IBM-Purdue & 0.2817 & 0.4942 & 0.631 \\
Visym Labs & 0.2835 & 0.4620 & 0.721 \\
UMD-Columbia & 0.3055 & 0.4716 & 0.516 \\
UMCMU & 0.3236 & 0.5297 & 0.464 \\
Purdue & 0.3327 & 0.5853 & 0.131 \\
MINDS_JHU & 0.4834 & 0.6649 & 0.967 \\
BUPT-MCPRL & 0.7985 & 0.9281 & 0.123 \\
\hline
\end{tabular}

In which, $[I]$ represents all input instances, and $[n]$ represent all target activities. Compared with vanilla BCE loss, we found wBCE loss can significantly improve the final performance on internal validation set.

Furthermore, we tried multiple action recognition modules and made late fusion action-wisely according to the results on the validation set. We found each classifier does show superiority on certain actions. Through the feedback from the online leaderboard, such fusion strategy can improve the final performance with noticeable margins.

\subsection{Activity Deduplication}

Overlapping Instances As the system generates overlapping proposals, it could have duplicate predictions for some of the proposals. This would result in a large amount of false alarms unless we deduplicate them. Figure 3 is a diagram for our deduplication algorithm which applies to each activity type with all proposals:

1. Split the overlapping cubes of duration $D_{\text {prop }}$ and stride $S_{\text {prop }}$ into non-overlapping cubes of duration $S_{\text {prop }}$. An output cube relies on all original cubes in the temporal window, with an averaged score and an intersected bounding box.

2. Merge the non-overlapping cubes of duration $S_{\text {prop }}$ back into $\left\lfloor\frac{D_{\text {prop }}}{S_{\text {prop }}}\right\rfloor$ groups of non-overlapping cubes of duration $D_{\text {prop }}$. An output cube is merged from $\left\lfloor\frac{D_{\text {prop }}}{S_{\text {prop }}}\right\rfloor$ cubes with an averaged score and the union of bounding boxes.

3. Select the group where the maximum score resides.

The deduplication algorithm performs an interpolation upon the overlapping cubes. Each group in step 3 contains information from every classification results, maximizing the information utilization.

Adjacent Instances The above deduplication process only transforms overlapping instances to non-overlapping instances with the same duration. This would be sufficient under the Loosened Setting, where multiple predictions are allowed for each activity. No threshold would be needed to truncate low-confidence predictions as this happens automatically during the ground-truth matching process.

However, for the Strict Setting, we need to further merge adjacent cubes into integrate instances. Currently we adopt a simple yet effective algorithm, by simply merging adjacent cubes where all of them have confidence score above $S_{m e r g}$. The merged instance needs to be longer than $L_{m e r g}$ to be kept in the final output.

\section{Experiments}

\subsection{Implementation Details}

In Argus++, we apply Mask R-CNN [8] with a ResNet101 [9] backbone from Detectron2 [26] pre-trained on the Microsoft COCO dataset [12] as the object detector, with 
Table 3. NIST ActEV'21 SDL Unknown Facility Evaluation

\begin{tabular}{lccc}
\hline System/Team & $n A U D C @ 0.2 T_{f a} \downarrow$ & Mean $P_{\text {miss }} @ 0.02 T_{f a} \downarrow$ & Relative Processing Time \\
\hline Argus++ (Ours) & $\mathbf{0 . 3 3 3 0}$ & $\underline{0.5438}$ & 0.776 \\
UCF & $\underline{0.3518}$ & $\mathbf{0 . 5 3 7 2}$ & 0.684 \\
IBM-Purdue & 0.3533 & 0.5531 & 0.575 \\
Visym Labs & 0.3762 & 0.5559 & 1.027 \\
UMD & 0.3898 & 0.5938 & 0.515 \\
UMD-Columbia & 0.4002 & 0.5975 & 0.520 \\
UMCMU & 0.4922 & 0.6861 & 0.614 \\
Purdue & 0.4942 & 0.7294 & 0.239 \\
MINDS_JHU & 0.6343 & 0.7791 & 0.898 \\
\hline
\end{tabular}

Table 4. NIST TRECVID 2021 ActEV Evaluation [1 30]

\begin{tabular}{lccc}
\hline System/Team & $n A U D C @ 0.2 T_{f a} \downarrow$ & Mean $P_{\text {miss }} @ 0.15 T_{f a} \downarrow$ & Mean $w P_{\text {miss }} @ 0.15 R_{f a} \downarrow$ \\
\hline Argus++ (Ours) & $\mathbf{0 . 3 9 6 0 7}$ & $\mathbf{0 . 3 0 6 2 2}$ & $\underline{0.81080}$ \\
BUPT & $\underline{0.40853}$ & $\underline{0.32489}$ & $\mathbf{0 . 7 9 7 9 8}$ \\
UCF & 0.43059 & 0.34080 & 0.86431 \\
M4D & 0.84658 & 0.79410 & 0.88521 \\
TokyoTech_AIST & 0.85159 & 0.81970 & 0.94897 \\
Team UEC & 0.96405 & 0.95035 & 0.95670 \\
\hline
\end{tabular}

$S_{\text {det }}=8$. Only person, vehicle, and traffic light classes are selected. For the tracking algorithm, we apply the work in [24] and reuse the region-of-interest from the ResNet backbone as in [28, 16].

The proposals are generated with $D_{\text {prop }}=64$ and $S_{\text {prop }}=16$. The labels are assigned with $S_{\text {high }}=0.5$ and $S_{\text {low }}=0$. The proposal filter is set with a tolerance of $P_{\text {pos }}=0.05$.

For activity classifiers, we adopted multiple state-of-theart models including $\mathrm{R}(2+1) \mathrm{D}$ [22], X3D [6], and Temporal Relocation Module (TRM) [17]. During training procedure, frames are cropped with jittering [23] and enlarged with $R_{e n l}=0.13$. For X3D and TRM, we trained modules with weights pre-trained on Kinetics [10]. For R(2+1)D modules, we trained modules with weighst pre-trained on IG65M [7]. We fused confidence scores from these models according to their performance on the validation set.

\subsection{Evaluation Protocols}

To measure the performance, efficiency, and generalizability of Argus++, we evaluate it across a series of public benchmarks. Argus++ is applied to NIST Activities in Extended Videos (ActEV) evaluations on MEVA [4] Unknown Facility, MEVA Known Facility, and VIRAT [15] settings for surveillance activity detection. With slight modifications, it is also tested in the ICCV 2021 ROAD challenge for the action detection task in autonomous driving.

In the NIST evaluations, the metrics [2] are designed in the Loosened Setting, where short-duration outputs are allowed and spatial alignment is ignored. The idea was that, after processed by the system, there will still be human reviewers to inspect the activity instances with the highest confidence scores for further usages. The performance is thus measured by the probability of miss detection $\left(P_{\text {miss }}\right)$ of activity instances within a time limit of all positive frames plus $T_{f a}$ of negative frames, where $T_{f a}$ is referred to as timebased false alarm rate. The major metric, $n A U D C @ 0.2 T_{f a}$, is an integration of $P_{\text {miss }}$ on $T_{f a} \in[0,0.2]$.

In the ROAD challenge, the Strict Setting is adopted by using the mean average precision (mAP) at $3 \mathrm{D}$ intersectionover-union (IoU) evaluation metric. This metric does exact bipartite matching between predictions and ground truth instances, with challenging localization precision requirements.

For metrics in the following tables, $\downarrow$ means lower is better and $\uparrow$ means higher is better. For each metric, the best value is bolded and the second best is underscored. For ongoing public evaluations, the result snapshot at 11/01/2021 is presented.

\subsection{ActEV Sequestered-Data Evaluation}

ActEV Sequestered Data Leaderboards (SDL) are platforms where a system is submitted to run on NIST's evaluation servers. This submission format prevents access to the test data and measures the processing time with unified hardware platform 3 For these evaluations, Argus ++ was trained on MEVA, a large-scale surveillance video dataset with activity annotations of 37 types. We used 1946 videos

3 https://actev.nist.gov/pub/Phase3_ActEV_2021_ SDL_EvaluationPlan_20210803.pdf 
Table 5. NIST TRECVID 2020 ActEV Evaluation [2] 29]

\begin{tabular}{lccc}
\hline System/Team & $n A U D C @ 0.2 T_{f a} \downarrow$ & Mean $P_{m i s s} @ 0.15 T_{f a} \downarrow$ & Mean $w P_{m i s s} @ 0.15 R_{f a} \downarrow$ \\
\hline Argus++ (Ours) & $\mathbf{0 . 4 2 3 0 7}$ & $\mathbf{0 . 3 3 2 4 1}$ & $\mathbf{0 . 8 0 9 6 5}$ \\
UCF & $\underline{0.54830}$ & 0.50285 & 0.83621 \\
BUPT-MCPRL & 0.55515 & $\underline{0.48779}$ & 0.84519 \\
TokyoTech_AIST & 0.79753 & 0.75502 & 0.87889 \\
CERTH-ITI & 0.86576 & 0.84454 & 0.88237 \\
Team UEC & 0.95168 & 0.95329 & 0.98300 \\
Kindai_Kobe & 0.96267 & 0.95204 & 0.93905 \\
\hline
\end{tabular}

in its training release drop 11 as the training set and 257 videos in its $\mathrm{KF} 1$ release as validation set. The optimization target is reaching better performance within $1 \mathrm{x}$ real-time.

Table 1 shows the published results from CVPR 2021 ActivityNet Challenge ActEV SDL Unknown Facility evaluation, where Argus++ demonstrated around 20\% advantage in $n A U D C @ 0.2 T_{f a}$ over runner-up system. The test set of unknown facility is captured with a different setting from MEVA, which challenges the generalization of action detection models. Table 2 shows the ongoing NIST ActEV'21 SDL Known Facility leaderboard, where Argus ++ shows over $40 \%$ advantage in $n A U D C @ 0.2 T_{f a}$. The test set of known facility shares a similar distribution with MEVA, where our system learns well and is getting nearer for realworld usages. Table 3 shows the ongoing NIST ActEV'21 SDL Unknown Facility leaderboard continued from ActivityNet, where Argus ++ still holds the leading position with over 5\% advantage in $n A U D C @ 0.2 T_{f a}$.

\subsection{ActEV Self-Reported Evaluation}

ActEV self-reported evaluations are where only results are submitted and test data is accessible. This currently includes the annual TRECVID ActEV evaluations on VIRAT. For TRECVID, we use the official splits of VIRAT for training and validation.

Table 4 and 5] shows the leaderboard of 2020 and 2021 NIST TRECVID ActEV Challenge. In 2020, our systems is $22.8 \%$ better in $n A U D C @ 0.2 T_{f a}, 33.8 \%$ better in Mean $P_{\text {miss }} @ 0.15 T_{f a}$, and 3.5\% better in Mean$w P_{\text {miss }} @ 0.15 R_{f a}$ than the runner-up. Although the other competitors improved significantly in 2021, our system still holds the first place with noticeable margins.

\subsection{ROAD Challenge}

Different from previous surveillance action detection benchmarks, the videos of ROAD Challenge[14] are gathered from the point of view of autonomous vehicles. It contains $122 \mathrm{~K}$ frames from 22 annotated videos, where each video is 8 minutes long on average. Totally $7 \mathrm{~K}$ tubes of individual agents are included and each tube consists on average of approximately 80 bounding boxes linked over time.

Table 6 shows the performance of our system with other competitors. Our system ranks the first with $20 \%$ average mAP. Although the performance is still far from satisfying in this Strict Setting, it demonstrates the capability of Argus ++ in adapting to precise 3D localization and moving camera view points.

\subsection{Ablation Study}

Coverage of Proposal Formats We analyze the coverage of dense spatio-temporal proposals and determines the best hyper-parameters for the proposal format. By directly use ground truth cubes as proposals, we estimate the upper bound performance of both overlapping and non-overlapping proposal formats on VIRAT validation set. The results are shown in Table 8, where non-overlapping proposals shows at least $6.7 \%$ systematic errors while overlapping proposals with duration 64 and stride 16 only has $1.3 \%$.

Performance of Proposal Filtering We examine the quality of the proposals with and without the filter, as shown in Table 9 and 7. With the proposal evaluation procedure introduced in Section 3.4, the proposals are further filtered by IoU with reference and coverage of reference at levels from $0,0.1$, to 0.9 to calculate partial results.

With the dense cube proposals, the best $n A U D C @ 0.2 T_{f a}$ we can achieve with a ideal classifier is 0.08 , as indicated in the IoU $\geq 0$ column. The IoU and reference coverage bounded scores are used to measure the spatial matching quality of proposals, as the $n A U D C @ 0.2 T_{f a}$ does not consider spatial alignments. We can see that even with a condition of IoU $\geq 0.5$, our proposal can achieve up to 0.15 , which indicates the spatial preciseness. The proposal filter is also proved effective, which removed $70 \%$ of original proposals without dropping the recall level.

The effect of the proposal filter is also evaluate on the SDL, as shown in Table 10. It not only reduces processing time from 0.925 to 0.582 , but also improves $n A U D C @ 0.2 T_{f a}$ due to reduced false alarms.

4 https://eval.ai/web/challenges/challenge-page/ $1059 /$ leaderboard/2748 
Table 6. ICCV 2021 ROAD Challenge Action Detection ${ }^{4}$

\begin{tabular}{lcccc}
\hline System/Team & Action@0.1 $\uparrow$ & Action@0.2 $\uparrow$ & Action@0.5 $\uparrow$ & Average $\uparrow$ \\
\hline Argus++ (Ours) & $\mathbf{2 8 . 5 4}$ & $\mathbf{2 5 . 6 3}$ & 6.98 & $\mathbf{2 0 . 3 8}$ \\
THE IFY & $\underline{28.15}$ & $\underline{20.97}$ & 6.58 & $\underline{18.57}$ \\
YAAAHO & 26.81 & 20.40 & $\underline{7.02}$ & 18.07 \\
hyj & 26.52 & 20.32 & $\mathbf{7 . 0 5}$ & 17.97 \\
3D RetinaNet [21] & 25.70 & 19.40 & 6.47 & 17.19 \\
LeeC & 13.64 & 9.89 & 2.23 & 8.59 \\
\hline
\end{tabular}

Table 7. Proposal Quality Metrics on VIRAT Validation Set

\begin{tabular}{lcccccc}
\hline$n A U D C @ 0.2 T_{f a}$ & \multicolumn{3}{c}{ IoU } & \multicolumn{4}{c}{ Reference Coverage } \\
Threshold & Average & $\geq 0$ & $\geq 0.5$ & Average & $\geq 0.5$ & $\geq 0.9$ \\
\hline Unfiltered Proposals & 0.2358 & 0.0772 & 0.1518 & 0.1562 & 0.1125 & 0.4211 \\
Filtered Proposals & 0.2352 & 0.0772 & 0.1469 & 0.1563 & 0.1099 & 0.4280 \\
\hline
\end{tabular}

Table 8. Lower Bounds of $n A U D C @ 0.2 T_{f a}$ on VIRAT Validation Set with different proposal formats. Italic values are nonoverlapping proposals while the others are overlapping proposals. Duration and stride are in the unit of frames.

\begin{tabular}{ccccc}
\hline Duration / Stride & 16 & 32 & 64 & 96 \\
\hline 32 & 0.0705 & 0.1208 & - & - \\
64 & $\mathbf{0 . 0 1 2 7}$ & 0.0621 & 0.0673 & - \\
96 & 0.0275 & 0.0504 & - & 0.0688 \\
\hline
\end{tabular}

Table 9. Statistics of Proposals on VIRAT Validation Set

\begin{tabular}{lcc}
\hline Name & Unfiltered & Filtered \\
\hline Number of Proposals & 211271 & 62831 \\
Positive rate & 0.1704 & $\mathbf{0 . 5 2 0 4}$ \\
Rate of unique label & 0.4558 & 0.4415 \\
Rate of two labels & 0.4127 & 0.4252 \\
Rate of three labels & 0.1017 & 0.1060 \\
\hline
\end{tabular}

Table 10. Proposal Filter on NIST ActEV'21 SDL Unknown Facility Micro Set

\begin{tabular}{lcc}
\hline Proposal Filter & $n A U D C @ 0.2 T_{f a} \downarrow$ & Processing Time \\
\hline Enabled & $\mathbf{0 . 4 8 2 2}$ & 0.582 \\
Disabled & 0.5176 & 0.925 \\
\hline
\end{tabular}

\section{Conclusion}

In this work, we proposed Argus++, a robust real-time activity detection system for analyzing unconstrained video streams. We introduced overlapping spatio-temporal cubes as an intermediate concept of activity proposals to ensure coverage and completeness of activity detection through over-sampling. The proposed system is able to process unconstrained videos with robust performance across multiple scenarios and real-time effiency on consumer-level hardware. Extensive experiments on different surveillance and driving scenarios demonstrated its superior performance in a series of activity detection benchmarks, including CVPR ActivityNet ActEV 2021, NIST ActEV SDL UF/KF, TRECVID ActEV 2020/2021, and ICCV ROAD 2021.

Future works are suggested to focus on extending the current system to more applications, such as action detection in UAV captured videos, first-person human activity understanding, etc. The proposed system could also be extended to end-to-end frameworks for better performance.

\section{Acknowledgements}

This research is supported in part by the Intelligence Advanced Research Projects Activity (IARPA) via Department of Interior/Interior Business Center (DOI/IBC) contract number D17PC00340. This research is supported in part through the financial assistance award 60NANB17D156 from U.S. Department of Commerce, National Institute of Standards and Technology. This project is funded in part by Carnegie Mellon University's Mobility21 National University Transportation Center, which is sponsored by the US Department of Transportation. 


\section{References}

[1] George Awad, Asad A. Butt, Keith Curtis, Jonathan Fiscus, Afzal Godil, Yooyoung Lee, Andrew Delgado, Jesse Zhang, Eliot Godard, Baptiste Chocot, Lukas Diduch, Jeffrey Liu, Yvette Graham, Gareth J. F. Jones, , and Georges Quénot. Evaluating multiple video understanding and retrieval tasks at trecvid 2021. In Proceedings of TRECVID 2021. NIST, USA, 2021.

[2] George Awad, Asad A. Butt, Keith Curtis, Jonathan Fiscus, Afzal Godil, Yooyoung Lee, Andrew Delgado, Jesse Zhang, Eliot Godard, Baptiste Chocot, Lukas Diduch, Jeffrey Liu, Alan F. Smeaton, Yvette Graham, Gareth J. F. Jones, Wessel Kraaij, and Georges Quenot. TRECVID 2020: A comprehensive campaign for evaluating video retrieval tasks across multiple application domains. arXiv:2104.13473 [cs], Apr. 2021.

[3] Xiaojun Chang, Wenhe Liu, Po-Yao Huang, Changlin Li, Fengda Zhu, Mingfei Han, Mingjie Li, Mengyuan Ma, Siyi $\mathrm{Hu}$, and Guoliang Kang. MMVG-INF-Etrol@ TRECVID 2019: Activities in Extended Video. In TREC Video Retrieval Evaluation, TRECVID, 2019.

[4] Kellie Corona, Katie Osterdahl, Roderic Collins, and Anthony Hoogs. MEVA: A Large-Scale Multiview, Multimodal Video Dataset for Activity Detection. In 2021 IEEE Winter Conference on Applications of Computer Vision (WACV), pages 1059-1067, Waikoloa, HI, USA, Jan. 2021. IEEE.

[5] Jifeng Dai, Yi Li, Kaiming He, and Jian Sun. R-fen: Object detection via region-based fully convolutional networks. In Advances in neural information processing systems, pages 379-387, 2016.

[6] Christoph Feichtenhofer. X3D: Expanding Architectures for Efficient Video Recognition. In 2020 IEEE/CVF Conference on Computer Vision and Pattern Recognition (CVPR), pages 200-210, Seattle, WA, USA, June 2020. IEEE.

[7] Deepti Ghadiyaram, Du Tran, and Dhruv Mahajan. LargeScale Weakly-Supervised Pre-Training for Video Action Recognition. In 2019 IEEE/CVF Conference on Computer Vision and Pattern Recognition (CVPR), pages 12038-12047, Long Beach, CA, USA, June 2019. IEEE.

[8] Kaiming He, Georgia Gkioxari, Piotr Dollár, and Ross Girshick. Mask R-CNN. IEEE Transactions on Pattern Analysis and Machine Intelligence, 42(2):386-397, Feb. 2020.

[9] Kaiming He, Xiangyu Zhang, Shaoqing Ren, and Jian Sun. Deep Residual Learning for Image Recognition. In 2016 IEEE Conference on Computer Vision and Pattern Recognition (CVPR), pages 770-778, Las Vegas, NV, USA, June 2016. IEEE.

[10] Will Kay, Joao Carreira, Karen Simonyan, Brian Zhang, Chloe Hillier, Sudheendra Vijayanarasimhan, Fabio Viola, Tim Green, Trevor Back, Paul Natsev, Mustafa Suleyman, and Andrew Zisserman. The Kinetics Human Action Video Dataset. arXiv:1705.06950 [cs], May 2017.

[11] Ji Lin, Chuang Gan, and Song Han. TSM: Temporal Shift Module for Efficient Video Understanding. In 2019 IEEE/CVF International Conference on Computer Vision (ICCV), pages 7082-7092, Seoul, Korea (South), Oct. 2019. IEEE.
[12] Tsung-Yi Lin, Michael Maire, Serge Belongie, James Hays, Pietro Perona, Deva Ramanan, Piotr Dollár, and C Lawrence Zitnick. Microsoft coco: Common objects in context. In European conference on computer vision, pages 740-755. Springer, 2014.

[13] Wenhe Liu, Guoliang Kang, Po-Yao Huang, Xiaojun Chang, Lijun Yu, Yijun Qian, Junwei Liang, Liangke Gui, Jing Wen, Peng Chen, and Alexander G. Hauptmann. Argus: Efficient Activity Detection System for Extended Video Analysis. In 2020 IEEE Winter Applications of Computer Vision Workshops (WACVW), pages 126-133, Snowmass Village, CO, USA, Mar. 2020. IEEE.

[14] Will Maddern, Geoff Pascoe, Chris Linegar, and Paul Newman. 1 Year, 1000km: The Oxford RobotCar Dataset. The International Journal of Robotics Research (IJRR), 36(1):315, 2017.

[15] Sangmin Oh, Anthony Hoogs, Amitha Perera, Naresh Cuntoor, Chia-Chih Chen, Jong Taek Lee, Saurajit Mukherjee, J. K. Aggarwal, Hyungtae Lee, Larry Davis, Eran Swears, Xioyang Wang, Qiang Ji, Kishore Reddy, Mubarak Shah, Carl Vondrick, Hamed Pirsiavash, Deva Ramanan, Jenny Yuen, Antonio Torralba, Bi Song, Anesco Fong, Amit Roy-Chowdhury, and Mita Desai. A large-scale benchmark dataset for event recognition in surveillance video. In CVPR 2011, pages 31533160, June 2011. ISSN: 1063-6919.

[16] Yijun Qian, Lijun Yu, Wenhe Liu, and Alexander G. Hauptmann. ELECTRICITY: An Efficient Multi-camera Vehicle Tracking System for Intelligent City. In 2020 IEEE/CVF Conference on Computer Vision and Pattern Recognition Workshops (CVPRW), pages 2511-2519, Seattle, WA, USA, June 2020. IEEE.

[17] Yijun Qian, Lijun Yu, Wenhe Liu, and Alexander G. Hauptmann. Trm: Temporal relocation module for video recognition. In Proceedings of the IEEE Winter Conference on Applications of Computer Vision Workshops, 2022.

[18] Yijun Qian, Lijun Yu, Wenhe Liu, Guoliang Kang, and Alexander G. Hauptmann. Adaptive Feature Aggregation for Video Object Detection. In 2020 IEEE Winter Applications of Computer Vision Workshops (WACVW), pages 143-147, Snowmass Village, CO, USA, Mar. 2020. IEEE.

[19] Shaoqing Ren, Kaiming He, Ross Girshick, and Jian Sun. Faster R-CNN: Towards Real-Time Object Detection with Region Proposal Networks. In Advances in Neural Information Processing Systems, volume 28. Curran Associates, Inc., 2015.

[20] Mamshad Nayeem Rizve, Ugur Demir, Praveen Tirupattur, Aayush Jung Rana, Kevin Duarte, Ishan R Dave, Yogesh S Rawat, and Mubarak Shah. Gabriella: An Online System for Real-Time Activity Detection in Untrimmed Security Videos. In 2020 25th International Conference on Pattern Recognition (ICPR), pages 4237-4244, Jan. 2021. ISSN: 1051-4651.

[21] Gurkirt Singh, Stephen Akrigg, Manuele Di Maio, Valentina Fontana, Reza Javanmard Alitappeh, Suman Saha, Kossar Jeddisaravi, Farzad Yousefi, Jacob Culley, Tom Nicholson, Jordan Omokeowa, Salman Khan, Stanislao Grazioso, Andrew Bradley, Giuseppe Di Gironimo, and Fabio Cuzzolin. ROAD: The ROad event Awareness Dataset for Autonomous Driving. arXiv:2102.11585 [cs], Feb. 2021. 
[22] Du Tran, Heng Wang, Lorenzo Torresani, Jamie Ray, Yann LeCun, and Manohar Paluri. A Closer Look at Spatiotemporal Convolutions for Action Recognition. In 2018 IEEE/CVF Conference on Computer Vision and Pattern Recognition, pages 6450-6459, Salt Lake City, UT, June 2018. IEEE.

[23] Limin Wang, Yuanjun Xiong, Zhe Wang, Yu Qiao, Dahua Lin, Xiaoou Tang, and Luc Van Gool. Temporal Segment Networks: Towards Good Practices for Deep Action Recognition. In Bastian Leibe, Jiri Matas, Nicu Sebe, and Max Welling, editors, Computer Vision - ECCV 2016, Lecture Notes in Computer Science, pages 20-36, Cham, 2016. Springer International Publishing.

[24] Zhongdao Wang, Liang Zheng, Yixuan Liu, Yali Li, and Shengjin Wang. Towards Real-Time Multi-Object Tracking. In Andrea Vedaldi, Horst Bischof, Thomas Brox, and Jan-Michael Frahm, editors, Computer Vision - ECCV 2020, volume 12356, pages 107-122. Springer International Publishing, Cham, 2020. Series Title: Lecture Notes in Computer Science.

[25] Nicolai Wojke, Alex Bewley, and Dietrich Paulus. Simple online and realtime tracking with a deep association metric. In 2017 IEEE International Conference on Image Processing (ICIP), pages 3645-3649. IEEE, 2017.

[26] Yuxin Wu, Alexander Kirillov, Francisco Massa, Wan-Yen Lo, and Ross Girshick. Detectron2. https://github. com/facebookresearch/detectron2, 2019.

[27] Lijun Yu, Peng Chen, Wenhe Liu, Guoliang Kang, and Alexander G. Hauptmann. Training-free Monocular 3D Event Detection System for Traffic Surveillance. In 2019 IEEE International Conference on Big Data (Big Data), pages 38383843, Dec. 2019.

[28] Lijun Yu, Qianyu Feng, Yijun Qian, Wenhe Liu, and Alexander G. Hauptmann. Zero-VIRUS * : Zero-shot Vehicle Route Understanding System for Intelligent Transportation. In 2020 IEEE/CVF Conference on Computer Vision and Pattern Recognition Workshops (CVPRW), pages 2534-2543, Seattle, WA, USA, June 2020. IEEE.

[29] Lijun Yu, Yijun Qian, Wenhe Liu, and Alexander G Hauptmann. CMU Informedia at TRECVID 2020: Activity Detection with Dense Spatio-temporal Proposals. In TREC Video Retrieval Evaluation, TRECVID, page 9, 2020.

[30] Lijun Yu, Yijun Qian, Wenhe Liu, and Alexander G Hauptmann. CMU Informedia at TRECVID 2021: Activity Detection with Argus++. In TREC Video Retrieval Evaluation, TRECVID, 2021.

[31] Lijun Yu, Dawei Zhang, Xiangqun Chen, and Alexander Hauptmann. Traffic Danger Recognition With Surveillance Cameras Without Training Data. In 2018 15th IEEE International Conference on Advanced Video and Signal Based Surveillance (AVSS), pages 1-6, Nov. 2018.

[32] Xizhou Zhu, Yujie Wang, Jifeng Dai, Lu Yuan, and Yichen Wei. Flow-Guided Feature Aggregation for Video Object Detection. In 2017 IEEE International Conference on Computer Vision (ICCV), pages 408-417, Venice, Oct. 2017. IEEE. 